\title{
Recurrent SKIL-activating rearrangements in ETS-negative prostate cancer
}

\author{
Matti Annala ${ }^{1,2, *}$, Kati Kivinummi ${ }^{1,2, *}$, Joonas Tuominen ${ }^{1,3}$, Serdar Karakurt ${ }^{1,3}$, Kirsi \\ Granberg ${ }^{1,2}$, Leena Latonen ${ }^{1,3}$, Antti Ylipääa, ${ }^{1,2}$, Liisa Sjöblom ${ }^{1,3}$, Pekka Ruusuvuori ${ }^{1,2}$, \\ Outi Saramäki ${ }^{1,3}$, Kirsi M. Kaukoniemi ${ }^{1,3}$, Olli Yli-Harja ${ }^{2}$, Robert L. Vessella ${ }^{4}$, Teuvo \\ L.J. Tammela ${ }^{5}$, Wei Zhang ${ }^{6}$, Tapio Visakorpi ${ }^{1,3}$ and Matti Nykter ${ }^{1,2}$ \\ ${ }^{1}$ Institute of Biosciences and Medical Technology - BioMediTech, University of Tampere, Tampere, Finland \\ 2 Institute of Biosciences and Medical Technology - BioMediTech, Tampere University of Technology, Tampere, Finland \\ ${ }^{3}$ Fimlab Laboratories, Tampere University Hospital, Tampere, Finland \\ ${ }^{4}$ Department of Urology, University of Washington, Seattle, WA, USA \\ ${ }^{5}$ Department of Urology, Tampere University Hospital and Medical School, University of Tampere, Tampere, Finland \\ ${ }^{6}$ Department of Pathology, University of Texas M.D. Anderson Cancer Center, Houston, TX, USA \\ * These authors contributed equally to this work \\ Correspondence to: Matti Nykter, email: matti.nykter@uta.fi \\ Tapio Visakorpi, email: tapio.visakorpi@uta.fi
}

Keywords: prostate cancer, sequencing, fusion gene, SKIL

Received: October 27, $2014 \quad$ Accepted: January 15, $2015 \quad$ Published: January 31, 2015

This is an open-access article distributed under the terms of the Creative Commons Attribution License, which permits unrestricted use, distribution, and reproduction in any medium, provided the original author and source are credited.

\section{ABSTRACT}

Prostate cancer is the third most common cause of male cancer death in developed countries, and one of the most comprehensively characterized human cancers. Roughly $60 \%$ of prostate cancers harbor gene fusions that juxtapose ETSfamily transcription factors with androgen regulated promoters. A second subtype, characterized by SPINK1 overexpression, accounts for $15 \%$ of prostate cancers. Here we report the discovery of a new prostate cancer subtype characterized by rearrangements juxtaposing the SMAD inhibitor SKIL with androgen regulated promoters, leading to increased SKIL expression. SKIL fusions were found in 6 of $540(1.1 \%)$ prostate cancers and 1 of $27(3.7 \%)$ cell lines and xenografts. 6 of 7 SKIL-positive cancers were negative for ETS overexpression, suggesting mutual exclusivity with ETS fusions. SKIL knockdown led to growth arrest in PC-3 and LNCaP cell line models of prostate cancer, and its overexpression led to increased invasiveness in RWPE-1 cells. The role of SKIL as a prostate cancer oncogene lends support to recent studies on the role of TGF- $\beta$ signaling as a rate-limiting step in prostate cancer progression. Our findings highlight SKIL as an oncogene and potential therapeutic target in 1-2\% of prostate cancers, amounting to an estimated 10,000 cancer diagnoses per year worldwide.

\section{INTRODUCTION}

Prostate cancer is diagnosed in over 900,000 men worldwide every year, making it the second most common cancer among men [1]. The standard-of-care for localized prostate cancer is radical prostatectomy or radiation therapy, whereas advanced tumors are treated with systemic therapies that inhibit androgen signaling
[2]. More specific drug targets and driver mutations have been sought through extensive genomic characterization efforts $[3,4]$. We now know that genomic rearrangement plays a major role in the onset of prostate cancer, with $60 \%$ of tumors harboring chromosomal rearrangements that juxtapose androgen regulated promoters with the ETS family transcription factors ERG, ETV1, ETV4 or FLII $[5,6]$. However, the products of these fusion genes have proven difficult to target with small molecule inhibitors. 
More recently, overexpression of the trypsin inhibitor SPINK1 was found to define a second prostate cancer subtype mutually exclusive with ETS overexpression [7]. Monoclonal SPINK1 antibodies have shown efficacy in preclinical models [8], suggesting that SPINK1 inhibition may prove a beneficial treatment strategy in the $15 \%$ of prostate cancers positive for SPINK1 overexpression. Recent studies have identified other alterations mutually exclusive with ETS fusions, including mutations in the SPOP gene [4], and deletions of the chromatin remodeling gene $C H D 1$ [9]. Despite these discoveries, a significant fraction of prostate cancers do not harbor any of the above alterations.

In addition to ETS fusions and associated events, genomic characterization studies have identified nonsynonymous mutations in TP53, MED12, and PTEN $[3,4,10]$, and gross deletions of the tumor suppressor genes PTEN, RB1 and TP53 [3,4,10]. More recently, a number of studies have highlighted the role of attenuated TGF- $\beta$ signaling in prostate cancer progression [11-13]. SMAD4, a critical component of the TGF- $\beta$ signaling cascade, is inactivated in a subset of advanced prostate cancers through promoter hypermethylation [14] or somatic mutation [10], and its expression is reduced in metastatic prostate cancer [11]. Mouse studies have shown that TGF- $\beta$ signaling inhibits progression of PTEN-null tumors, and that SMAD4 deletion can overcome this inhibition [11]. In pancreatic adenocarcinoma, biallelic inactivation of SMAD4 is observed in $50 \%$ of tumors [15].

In this study, we performed transcriptome and lowcoverage whole genome sequencing on 28 untreated and 13 castration resistant prostate cancers, and identified a new prostate cancer subtype characterized by activating rearrangements of the SMAD inhibitor SKIL.

\section{RESULTS}

\section{Sample acquisition and sequencing}

Fresh-frozen tissue from 12 benign prostatic hyperplasias (BPH), 28 untreated prostate cancers (PC), and 13 castration resistant prostate cancers (CRPC) was acquired from the Tampere University Hospital (Tampere, Finland). All samples contained a minimum of $70 \%$ cancerous or hyperplastic epithelial cells. PC samples were obtained by radical prostatectomy and locally recurrent CRPCs by transurethral resection of the prostate (Supplementary Table 1). Mean age at diagnosis was 60.8 years (range: 47.4-71.8) and mean PSA at diagnosis was $10.8 \mathrm{ng} / \mathrm{ml}$ (range: 3.5-48.1). Libraries were prepared for paired-end analysis on the Illumina HiSeq 2000. On average, we obtained 150 million paired end reads per sample from the low coverage whole genome sequencing, and 110 million paired end reads from the whole transcriptome sequencing (Supplementary Table 2).

\section{Discovery of recurrent SKIL-activating rearrangements}

Fusion events involving an ETS family transcription factor or SPINK1 overexpression were identified in 32 of 41 tumors based on transcriptome sequencing (Figure 1a, Supplementary Table 3). No SPOP mutations were identified in our cohort. We identified a novel TMPRSS2SKIL fusion gene in one CRPC sample (Figure 1AB, Supplementary Figure 1) and validated it using Sanger sequencing (Figure 1B) and fluorescence in situ hybridization (Figure 1C). The fusion merged the first three exons of TMPRSS 2 with full length SKIL and led to $S K I L$ overexpression due to the androgen regulated TMPRSS2 promoter (Figure 1D). SKIL encodes a SKIlike protein that inhibits TGF- $\beta$ signaling by binding to and disrupting the heteromeric SMAD complex [16]. To search for more positive cases, we screened 76 additional tumors (Supplementary Table 1) and $22 \mathrm{LuCaP}$ xenografts with qRT-PCR, and identified SKIL overexpression in one xenograft and one clinical sample. Transcriptome sequencing of these samples revealed a SLC45A3-SKIL fusion in LuCaP-77 and a MIPEP-SKIL fusion in the clinical sample, confirming SKIL as a recurrent 3' fusion partner in prostate cancer (Figure 1E-F). Analysis of transcriptome sequencing data from the Cancer Genome Atlas (TCGA) prostate adenocarcinoma project revealed additional SKIL-activating rearrangements in 4 of 423 samples, with concomitant SKIL overexpression (Figure 2A-B). In the Taylor et al. dataset, two ETS-negative samples (PCA0015 and PCA0056) exhibited outlier overexpression of $S K I L$, but were excluded from further analysis due to lack of sequencing data [3]. Interestingly, all 5 SKIL-positive clinical samples with clinical information (two TCGA samples lacked clinical data) contained a Gleason grade 5 component or represented metastatic prostate cancer, suggesting that SKIL-activating alterations may associate with high-grade prostate cancer.

None of the seven SKIL rearrangements disrupted the protein coding sequence of $S K I L$, suggesting that fulllength SKIL protein is necessary for oncogenic function. 6 of 7 SKIL rearrangements involved an androgen regulated promoter, indicating selection towards juxtapositions with highly active promoters. 6 of 7 fusion positive samples were negative for ETS overexpression, suggesting mutual exclusivity between SKIL and ETS rearrangements (p $=0.047$, Fisher's exact test). Sample TCGA-YL-A8SJ overexpressed both SKIL and ETVI (Figure 2A), although we found no reads supporting an ETV1 rearrangement in either the transcriptome or exome sequencing data for this sample.

In sample TCGA-HC-7211, the rearrangement between $A C P P$ and $S K I L$ had an unexpected structure, 


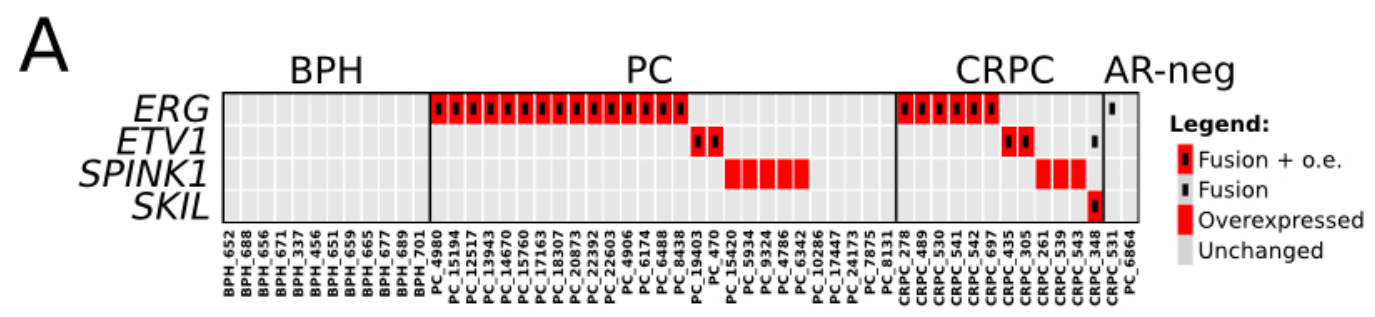

B
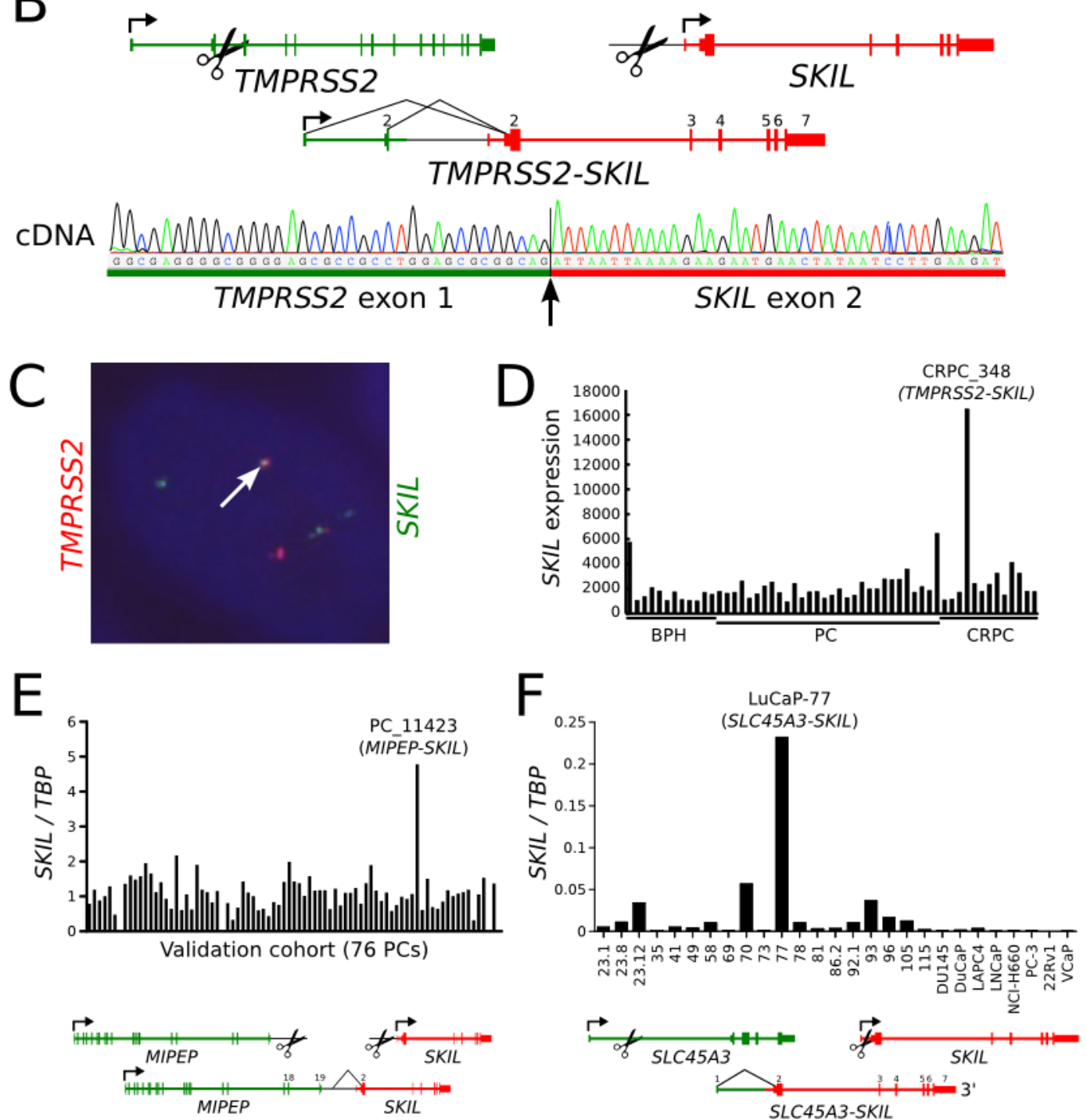

Figure 1: Recurrent $S K I L$-activating rearrangements in prostate cancer. (A) Matrix showing mutually exclusive overexpression of ERG, ETV1, ETV4, SPINK1, and SKIL in a transcriptome sequencing cohort of 41 prostate cancers. Red rectangles indicate overexpression, and black inner rectangles indicate fusion events. (B) Structure of the TMPRSS2-SKIL fusion gene identified in sample CRPC_348. Black lines indicate exon-exon junctions with transcriptome sequencing evidence. Fusion transcript was validated with Sanger sequencing from cDNA. (C) Fluorescence in situ hybridization validates the fusion at genomic level. One example of a fusion positive cell is shown. (D) SKIL expression in the transcriptome sequencing cohort of 41 prostate cancers and 12 BPHs. SKIL is strongly overexpressed in the TMPRSS2-SKIL positive sample. (E) SKIL expression was measured using qRT-PCR in a validation cohort of 76 prostatectomy samples. Sample PC_11423 exhibited SKIL overexpression and was found to contain a MIPEP-SKIL rearrangement by transcriptome sequencing. (F) SKIL expression was measured using qRT-PCR in LuCaP xenografts and cell line models of prostate cancer. Xenograft LuCaP-77 was found to contain an SLC45A3-SKIL rearrangement by transcriptome sequencing. 
with the first exon and promoter of $A C P P$ placed downstream of SKIL in antisense orientation (Figure 2B). Despite the non-canonical structure, the rearrangement led to strong overexpression of full-length SKIL (Figure 2A), possibly due to chromatin remodeling induced by the androgen regulated $A C P P$ promoter. The antisense promoter provoked expression of a spliced antisense transcript composed of cryptic exons located in SKIL introns (Supplementary Figure 2). Expression of the antisense transcript did not appear to interfere with $S K I L$ splicing, as sense transcripts were normally spliced.

\section{Level of nuclear SKIL protein is elevated in a fusion positive sample}

To determine whether fusion positive clinical samples also overexpressed SKIL at the protein level, we used a monoclonal antibody to perform immunohistochemistry on the fusion positive TURP sample CRPC_348 and 8 negative controls. The TURP sample exhibited strong nuclear and modest cytoplasmic staining for SKIL, while negative controls showed no staining or only weak cytoplasmic staining for SKIL (Figure 3A). We also showed the overexpression of SKIL at the RNA level in CRPC_348 using RNA in situ hybridization (Figure 3B).

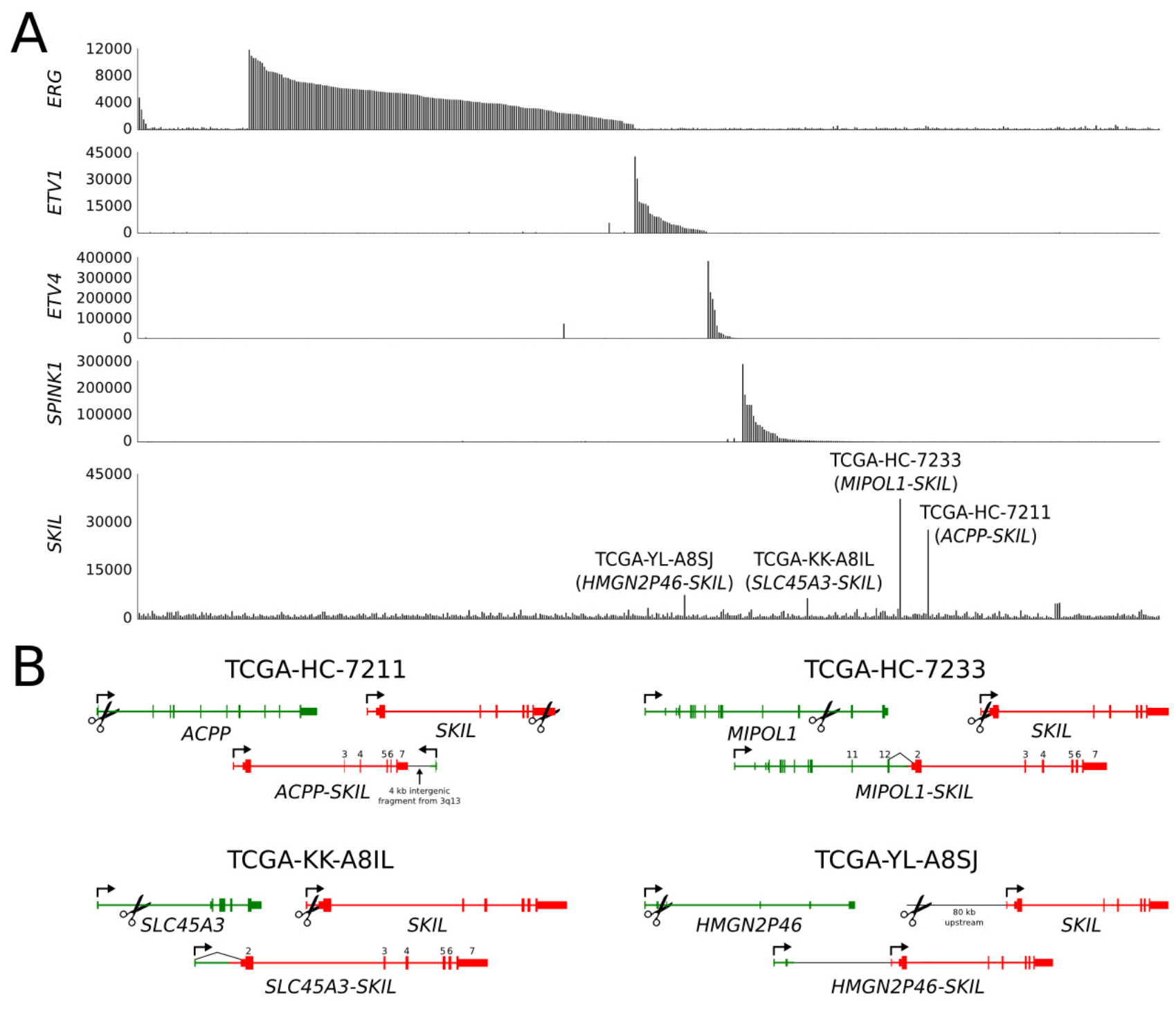

Figure 2: $S K I L$-activating rearrangements in the TCGA prostate adenocarcinoma sequencing cohort. (A) Barplot showing expression of ERG, ETV1, ETV4, SPINK1 and SKIL in TCGA samples. Four samples exhibited significant SKIL overexpression and were found to harbor SKIL-activating rearrangements. (B) Structures of the ACPP-SKIL, SLC45A3-SKIL, MIPOL1-SKIL and HMGN2P46-SKIL rearrangements. Black lines indicate exon-exon junctions with transcriptome sequencing evidence. 


\section{SKIL regulates proliferation and invasiveness of prostate cancer cells}

To better understand the biological role of SKIL in prostate cancer cells, we knocked SKIL down in PC-3 cells using two different siRNA (Figure 4A), and observed reduced cell growth (Figure 4B), invasiveness (Figure 4c), and colony formation (Figure 4D) relative to scrambled siRNA. The effect on cell growth was replicated in $\mathrm{LNCaP}$ cells (Figure 4E-F). To show that SKIL expression is truly androgen dependent in cells where SKIL is fused with androgen regulated promoters, we extracted $\mathrm{LuCaP}-77$ xenograft tissue (with an SLC45A3-SKIL fusion) from castrate and non-castrate mice 1,3 and 7 days postcastration. We then used qRT-PCR to quantify SKIL and $K L K 3$ (PSA) expression at each timepoint. Expression values were normalized against TATA-box binding protein $(T B P)$ and compared against non-castrate control mice. We observed a strong reduction in the expression of both $K L K 3$ and SKIL on day 7, indicating androgen regulated expression (Figure 4G).

Next, we created a SKIL overexpression model by transfecting immortalized prostate epithelial cells (RWPE1) with a pCI-Neo vector expressing hemagglutinin (HA) tagged SKIL [17]. Control RWPE-1 cells were transfected with an empty pCI-Neo vector (EV). In comparison to control cells, SKIL-transfected RWPE-1 cells exhibited higher expression of SKIL at both RNA and protein levels (Figure 5A-B), and greater invasive potential in a matrigel invasion assay $(n=3, p=0.044$, unpaired two-tailed t-test $)$ (Figure 5C). SKIL overexpression had no effect on the growth of RWPE-1 cells (data not shown).

\section{Other genomic alterations in the sequencing cohort}

A previous study has shown that combined deletion of SMAD4 and PTEN in mouse prostates leads to aggressive prostate cancer with $100 \%$ penetrance [11]. Therefore, we set out to check whether SKIL-rearranged cancers harbored concomitant PTEN deletions. PTEN expression was not aberrantly low in any of the 7 SKILpositive cases, and only 1 of 4 SKIL-positive cancers in the TCGA cohort showed evidence of PTEN deletion, suggesting that SKIL rearrangements do not require combined PTEN loss. Genes associated with $E R G$ overexpression, such as COL2A1, ALOX15, CRISP3, $B 3 G N T 6$ and TDRD1, were not overexpressed in SKILrearranged tumors.
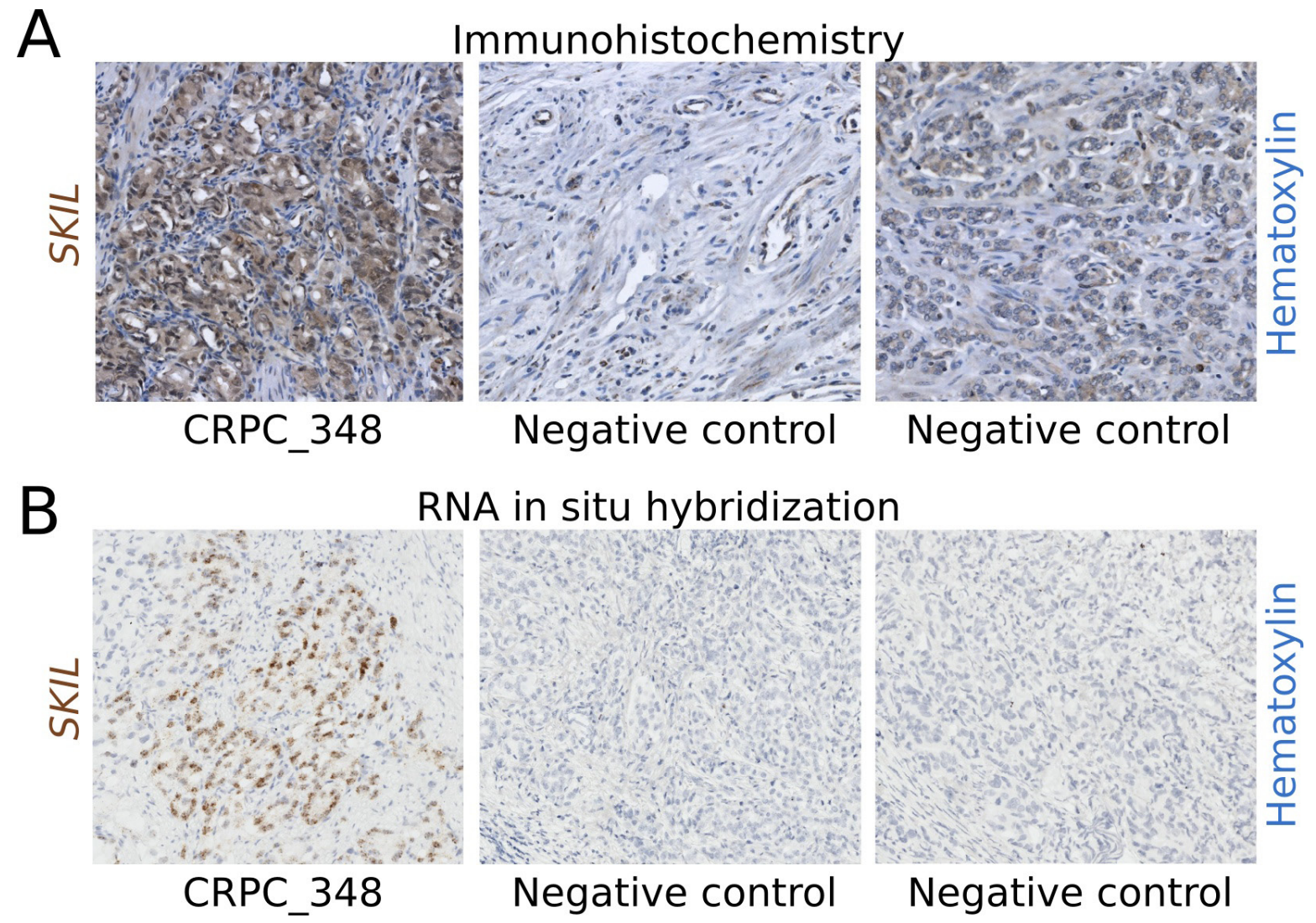

Figure 3: Immunohistochemistry and RNA in situ hybridization of SKIL in a SKIL-rearranged tumor. (A) Anti-SKIL staining of paraffin-embedded sections from the SKIL-rearranged TURP sample CRPC_348 and two representative prostatectomies negative for SKIL rearrangement showing no staining or weak cytoplasmic staining. (B) RNA in situ hybridization of CRPC_348 and two representative prostatectomies negative for SKIL rearrangement, with probes targeting SKIL mRNA. Nuclei were stained with hematoxylin. 
Expression analysis of the TGF- $\beta$ and BMP pathways (that both converge on SMAD4) revealed significantly reduced expression of TGF- $\beta$ and BMP ligands in both untreated and castration resistant prostate cancer (Figure 6, Supplementary Figure 3). Other genomic alterations in $S K I L$-positive tumors included TP53 mutation in CRPC 348, hemizygous TP53 deletion in TCGA-KK-A8IL and TCGA-YL-A8SJ, hemizygous PTEN deletion in TCGA-YL-A8SJ, hemizygous NKX31 deletion in TCGA-HC-7211 and TCGA-YL-A8SJ, and an MLL3 frameshift deletion mutation in CRPC_348 (Supplementary Table 4).

In addition to SKIL rearrangements, we found various other genomic alterations in our sequencing cohort (Figure 7, Supplementary Figure 4, Supplementary Table 5). Since the original sequencing did not include paired normal tissues, we used targeted sequencing on paired blood samples to filter out germline variants. The tumor suppressor TP53 was nonsynonymously mutated in 2 PCs and 5 CRPCs, with additional lossof-heterozygosity in 3 CRPCs. PTEN was disrupted by a stopgain mutation in one CRPC sample and deleted in eight other tumors. The AR negative tumor PC_6864 carried a $K R A S$ p.G12R mutation that is known to cause constitutive KRAS activation in cancers of the colon, pancreas and lungs, but is less common in prostate cancer [18]. Sample CRPC_489 harbored two distinct AKT1 mutations, one of which (p.E17K) has been associated with dysregulated tissue growth in the Proteus syndrome [19]. We also identified somatic mutations that altered the forkhead domain and the $\mathrm{N}$-terminal transactivation domain of the AR cofactor FOXA1. Four samples were positive for the $H O X B 13$ p.G84E germline variant that has been associated with prostate cancer susceptibility [20], including one homozygous sample. One AR-negative CRPC sample had acquired a DOT1L-HES6 fusion. We and others have shown that HES6 overexpression is sufficient to induce completely androgen independent growth in prostate cancer cells $[21,22]$. In both PC and CRPC, we identified frequent alterations in chromatin modifiers including nonsynonymous mutations in $\mathrm{CHD} 4$, $M L L 3, H D A C 5, K D M 5 B$ and $M B D 6$, and a homozygous deletion of $K D M 6 A$ in one sample (Figure 7).

\section{DISCUSSION}

SKIL (also known as SnoN) is a 684 amino acid

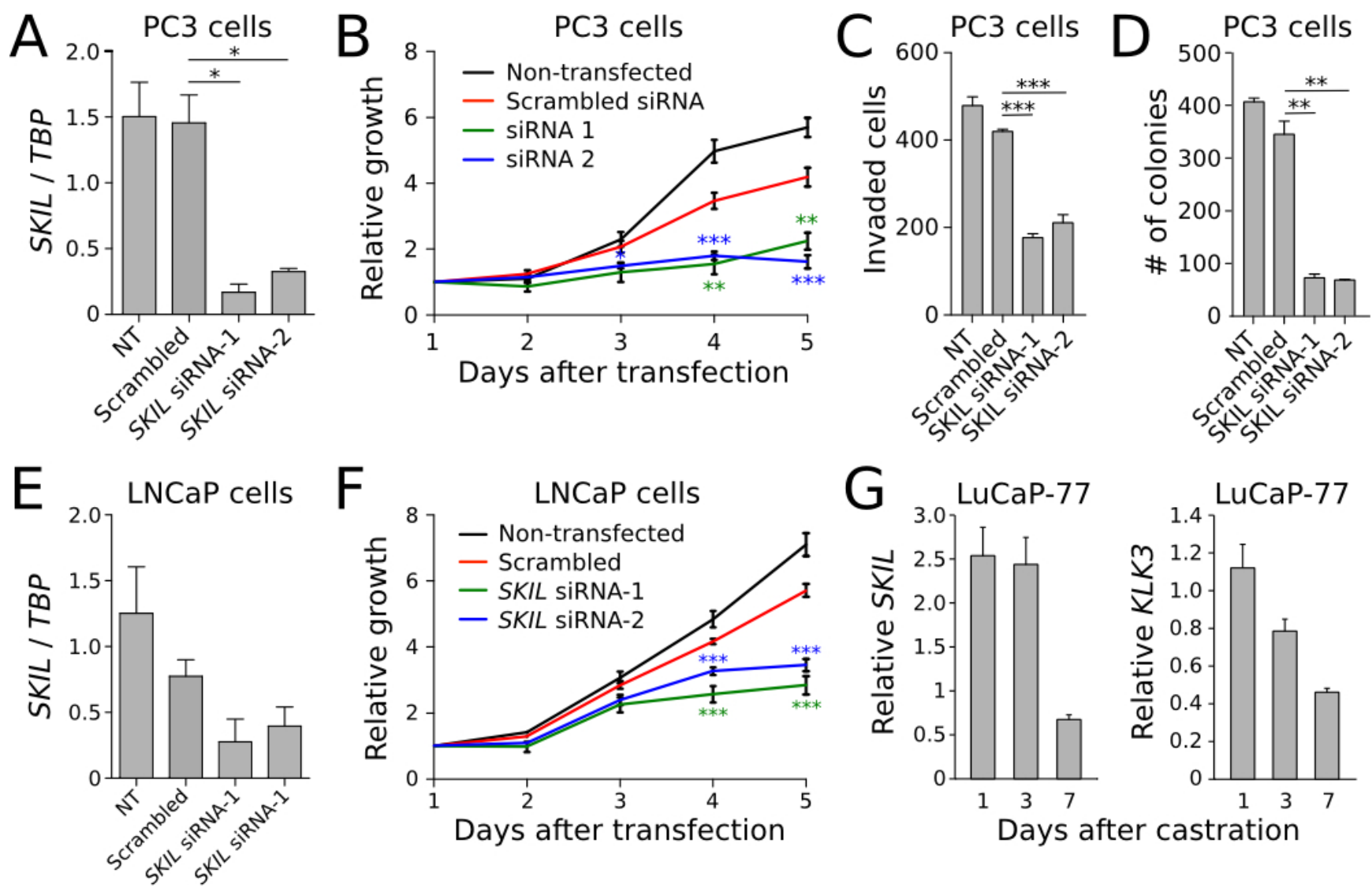

Figure 4: In vitro knockdown experiments on SKIL. (A) SKIL expression was silenced in PC3 cells using two siRNAs, resulting in (B) reduced growth $(\mathrm{n}=4),(\mathrm{C})$ invasion $(\mathrm{n}=4)$ and $(\mathrm{D})$ colony formation $(\mathrm{n}=2)$. (E) SKIL expression was silenced in LNCaP cells using two siRNAs, resulting in $(\mathrm{F})$ reduced growth $(\mathrm{n}=4)$. $(\mathrm{G})$ qRT-PCR time series of SKIL and PSA expression in castrate and non-castrate mice carrying LuCaP-77 xenografts $(\mathrm{n}=2)$. Error bars, s.e.m. with first-order error propagation; ${ }^{*} \mathrm{P}<0.05 ; * * \mathrm{P}<0.01 ; * * * \mathrm{P}<0.001$, unpaired two-tailed t-test. 

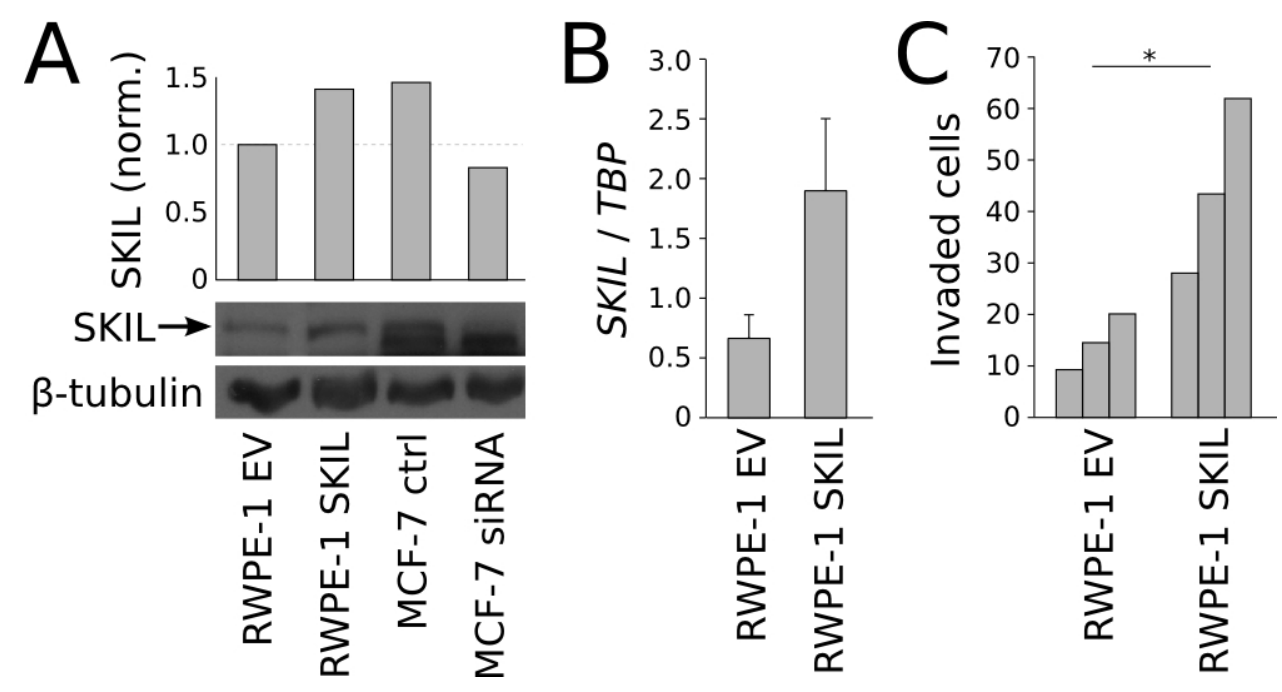

Figure 5: In vitro overexpression experiments on SKIL. (A) Anti-SKIL western blot showing increased SKIL protein in SKILtransfected RWPE-1 cells. MCF-7 cells transfected with SKIL or scrambled siRNA are used to validate the band. (B) qRT-PCR quantification of SKIL in RWPE-1 cells transfected with SKIL or empty vector. (C) Matrigen invasion assay on RWPE-1 cells transfected with SKIL or empty vector $(\mathrm{n}=3)$. Error bars, s.e.m. with first-order error propagation; $* \mathrm{P}<0.05 ; * * \mathrm{P}<0.01 ; * * * \mathrm{P}<0.001$, unpaired two-tailed t-test.

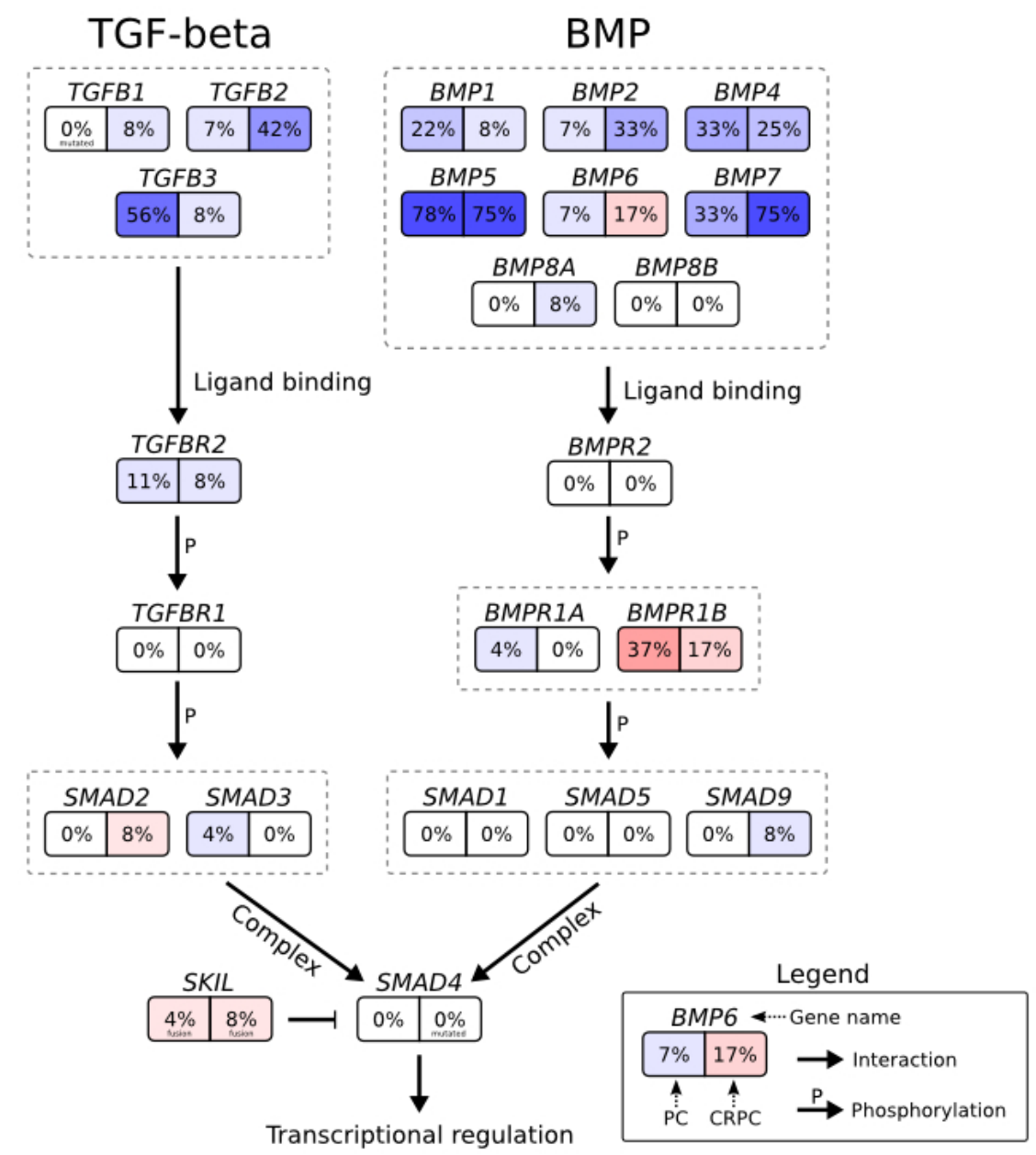

Figure 6: Genomic and transcriptomic changes in the context of the TGF- $\beta$ signaling pathway. Genes are shown as boxes with two halves: the left half shows the percentage of untreated prostate cancers with two-fold upregulation (red) or downregulation (blue) relative to $\mathrm{BPH}$, and the right half shows the same for castration resistant prostate cancers. Arrows indicate interactions between proteins or genes, the interaction type is written next to the arrow. 
nuclear protein that is ubiquitously expressed in human tissues [23,24] and shares its domain structure with SKI, a protein that was originally discovered through its similarity with the transforming component of the Sloan-Kettering Virus [25]. Overexpression of either SKI or SKIL in chicken embryo fibroblasts is sufficient to induce oncogenic transformation [26], and SKIL expression is elevated in many human cancers, including cancers of the skin, breast, colon and blood [27]. The 3 q26 locus is amplified in several cancer types, and SKIL (along with TLOC1) has been highlighted as the most potent oncogene in this region [28]. Both SKI and SKIL contain an 80 aa SAND-like domain that can bind with the MH2 domain of SMAD4 [29]. SMAD4 (coSmad) is an irreplaceable part of the heteromeric SMAD complexes that act as downstream mediators of TGF- $\beta$ signaling. These heteromeric complexes are formed when SMAD4 binds with one or more receptor SMADs such as SMAD2 and SMAD3 [29]. The complex then translocates to the nucleus and activates transcription of TGF- $\beta$ responsive genes. Binding of SKI/SKIL with the SMAD4 $\mathrm{MH} 2$ domain inhibits this transcriptional activation by preventing SMAD complex formation [29] or by recruitment of the nuclear co-repressor NCOR1 [16]. In addition to its binding with SMAD4, SKIL can also bind with the MH2 domains found in receptor SMADs through a domain located close to its N-terminal [16]. Neither SKI nor SKIL has been shown to directly bind DNA, despite both proteins containing a Dachshund homology domain that shares features with the forkhead/winged-helix family of DNA binding proteins [27].

In addition to the inhibitory role of SKIL on TGF- $\beta$ signaling, a recent study has proposed that SKIL may play a role in regulating epithelial-to-mesenchymal transition (EMT) by inducing expression of SNAI2 (SLUG), a master regulator of EMT [28]. The same study also found that SMAD4 knockdown increased the invasiveness of human mammary epithelial cells, while SKIL overexpression had no effect on cell growth, in agreement with our findings [28]. Another recent study has proposed that SKIL can interact with and promote the activity of estrogen receptor $\alpha$ in the nuclei of breast carcinoma cells. The interaction occurs via two highly conserved nuclear receptor binding LxxLL-like motifs in SKIL [30]. This

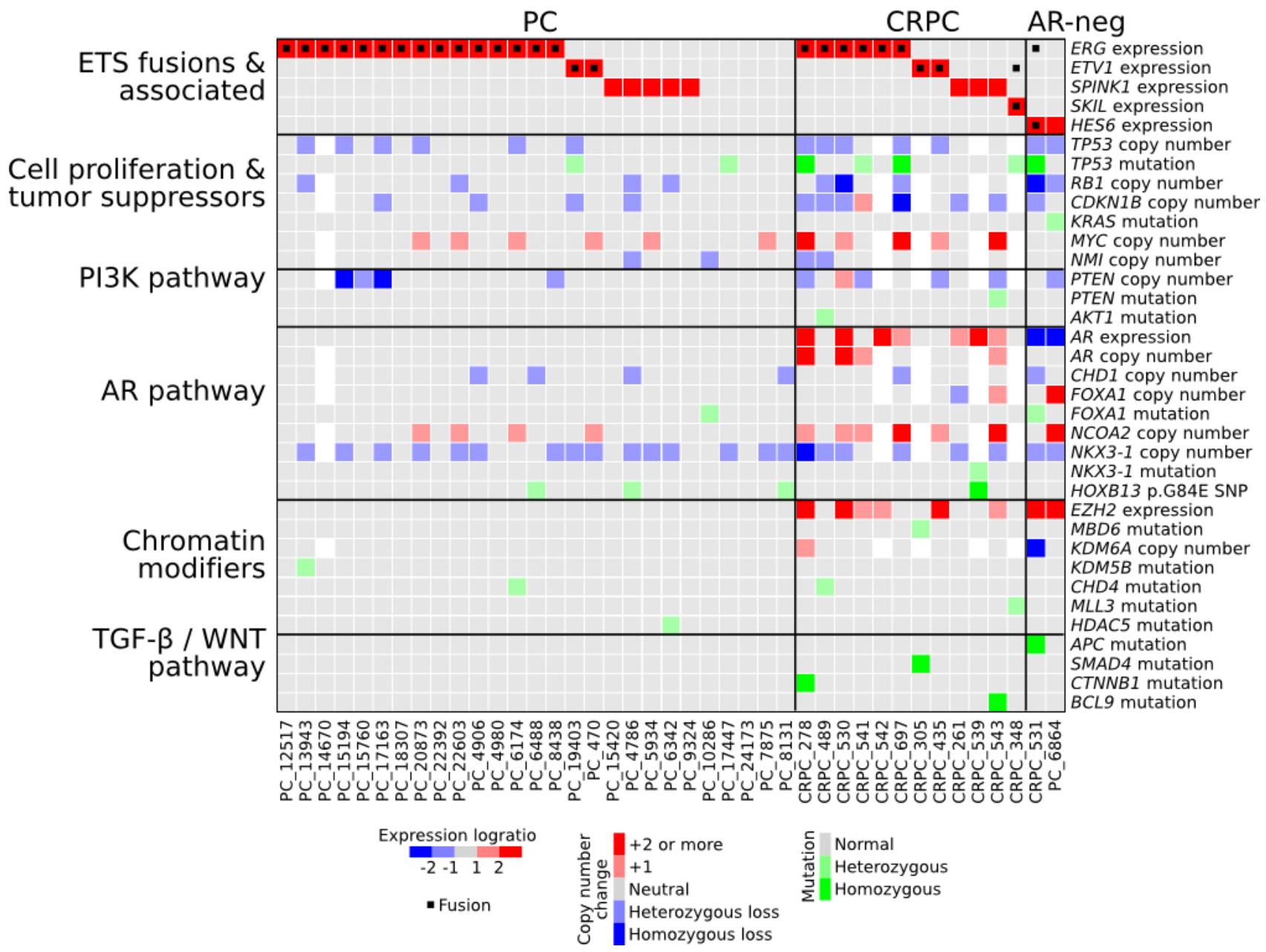

Figure 7: Characterization of genomic and transcriptomic changes in our prostate cancer sequencing cohort. Expression and copy number changes are shown in blue and red. Point mutations and indels affecting protein coding sequences are shown in green. White squares indicate missing data. 
finding is intriguing as it suggests a potential interaction between SKIL and androgen receptor, as some LxxLL motifs can bind with the ligand binding domain of AR [31].

The SKIL rearrangements reported in this paper occurred in both untreated and castration resistant prostate cancers, and involved the 5' partner genes TMPRSS2, SLC45A3, MIPOL1, ACPP, MIPEP and HMGN2P46. The androgen regulated genes TMPRSS2 and SLC45A3 are the most common $5^{\prime}$ partners involved in ETS fusions in prostate cancer [6]. MIPOL1 is another androgen regulated gene that is involved in MIPOL1-ETS rearrangements in prostate cancers [32]. Expression of $A C P P$ is androgen regulated and highly prostate specific among normal tissue types [33]. MIPEP is not generally considered androgen regulated or highly expressed in the prostate, but we observed high expression of both MIPEP and SKIL in the MIPEP-SKIL positive sample. HMGN2P46 is a pseudogene that is strongly expressed in AR-expressing prostate cancers but not expressed in AR-negative cancers in our cohort, suggesting an androgen regulated promoter. We conclude that six SKIL rearrangements involved an androgen regulated promoter, and one involved an otherwise highly active promoter.

The discovery of $S K I L$-activating rearrangements in both untreated and castration resistant prostate cancers suggests that SKIL rearrangements may represent an early event in prostate tumorigenesis. This hypothesis is supported by the fact that SKIL rearrangements appear to be mutually exclusive with ETS fusions, which represent a known early event in prostate cancer progression [34]. The complex structure of two rearrangements highlights the fact that rearrangements can affect gene expression in a clinically significant manner without disrupting the transcribed portion of a gene. As an example of this complexity, one $S K I L$-activating rearrangement in our study juxtaposed an active promoter to a position downstream of SKIL in opposite orientation, and still resulted in strongly elevated expression of full length SKIL transcript.

Based on the cohorts studied in this manuscript, we estimate that SKIL-activating rearrangements are found in $1-2 \%$ of diagnosed prostate cancers. Due to the high incidence of prostate cancer, this fraction translates to an estimated 10,000 diagnoses and 3,000 deaths caused by $S K I L$-positive prostate cancers per year worldwide [1]. Whether $S K I L$-positive prostate cancers differ in their clinical course from other prostate cancers remains to be evaluated in a larger study. Since 6 of 7 SKIL rearrangements involved androgen regulated promoters, we expect that existing treatment modalities based on androgen ablation will be effective at treating SKILrearranged cancers. Nonetheless, SKIL provides an intriguing new molecular target for personalized therapy, and highlights the role of TGF- $\beta$ signaling in prostate cancer progression.

\section{MATERIALS AND METHODS}

\section{Sequencing cohort}

Fresh-frozen tissue specimens from 12 benign prostate hyperplasias, 28 untreated prostate cancers, and 13 castration resistant prostate cancers were acquired from Tampere University Hospital (Tampere, Finland). Untreated prostate cancer samples were obtained by radical prostatectomy and locally recurrent CRPCs by transurethral resection of the prostate. Samples were snap-frozen and stored in liquid nitrogen. Histological evaluation and Gleason grading were performed by a pathologist based on hematoxylin/eosin-stained slides. All samples contained a minimum of $70 \%$ cancerous or hyperplastic cells. The use of clinical material was approved by the ethical committee of the Tampere University Hospital and the National Authority for Medicolegal Affairs. Written informed consent was obtained from the subjects.

\section{Validation cohort}

76 additional hormonally untreated PC prostatectomy samples were acquired from the Tampere University Hospital (Tampere, Finland). Samples were snap-frozen and stored in liquid nitrogen. Histological evaluation and Gleason grading were performed by a pathologist based on hematoxylin/eosin-stained slides. Samples were confirmed to contain a minimum of $70 \%$ cancerous or hyperplastic cells by hematoxylin-eosin staining. Mean age at diagnosis was 62.1 years (range: 47.4-71.8), mean PSA at diagnosis was 11.8 (range: 3.1551.5). The use of clinical material was approved by the ethical committee of the Tampere University Hospital and the National Authority for Medicolegal Affairs. Written informed consent was obtained from the subjects.

\section{Cell lines and xenografts}

Prostate cancer cell lines PC-3, LNCaP, DU145, 22Rv1 and immortalized prostate epithelial cell line RWPE-1 were obtained from American Type Cell Collection (Manassas, VA, USA). LAPC-4 cell line was kindly provided by Dr. Charles Sawyers (University of California at Los Angeles, Los Angeles, CA, USA), VCaP and DuCaP by Dr. Jack Schalken (Radboud University Nijmegen Medical Center, Nijmegen, the Netherlands), and EP156T by Dr. Varda Rotter (Weizmann Institute of Science, Rehovot, Israel). All cell lines were cultured under recommended conditions. 22 previously established LuCaP-series xenografts were provided by R.L.V. These xenografts have been derived from primary and metastatic 
human prostate cancer and are maintained in vivo [35].

\section{DNA and RNA extraction for sequencing cohort}

Fresh-frozen tissue blocks were cut into 10x20micrometer sections using a cryotome. RNA and DNA were isolated using an AllPrep RNA/DNA minikit (Qiagen, Valencia, CA, USA) according to manufacturer's protocol. For some samples, more total RNA was isolated using Trizol (Invitrogen, Carlsbad, CA, USA) extraction according to manufacturer's protocol. Three CRPC samples had RNA extracted using both Trizol and Qiagen AllPrep. The isolated RNA was quantified by based on $260 \mathrm{~nm}$ absorbance and its purity assessed by the $260 / 280$ nm ratio. Integrity was checked using Bioanalyzer (Agilent Technologies, Santa Clara, CA, USA). Reverse transcription of RNA to cDNA from clinical samples was carried out using SuperScript(TM)III (Invitrogen, Carlsbad, CA, USA) reverse transcriptase and AMV (Finnzymes, Espoo, Finland) for cell line samples with random hexamer primers according to manufacturer's instructions.

\section{Whole genome library construction and sequencing}

Genomic DNA was sonicated into 500 bp fragments using a Covaris E210. Overhangs were converted into blunt ends using T4 DNA polymerase and Klenow enzyme. An adenine was added to the 3' end of the blunt phosphorylated DNA fragments, and adapters were ligated on both ends. Ligated products were purified by agarose gel electrophoresis followed by QIA quick gel extraction, to remove residual free and self-ligated adaptors and to select properly sized templates for cluster generation. DNA fragments with adapters on both ends were amplified using two primers that annealed to the adapters. PCR products were checked and purified by agarose gel electrophoresis. The fragment size and molar concentration of each library was determined using Agilent 2100 Bioanalyzer and ABI Real-Time PCR System (StepOnePlus ${ }^{\mathrm{TM}}$ ), respectively. As mean fragment size increased to $622 \mathrm{bp}$ after adapter ligation, fragments between $600 \mathrm{bp}$ and 684 bp were selected. An Illumina Cluster Station was used to hybridize samples onto a flow cell and amplify them for sequencing on Illumina HiSeq ${ }^{\mathrm{TM}}$ 2000. Raw image files were processed by Illumina pipeline for base-calling with default parameters resulting in $90 \mathrm{bp}$ paired end reads. Reads with too many $\mathrm{N}$ bases $(>10 \%)$ or low base quality $(>50 \%$ bases with base quality $<5)$ were discarded. Library construction and sequencing was performed at the Beijing Genomics Institute (BGI), Hong Kong.

\section{Whole transcriptome library construction and sequencing}

Beads with Oligo(dT) were used to isolate poly(A) mRNA after collection of total RNA. Fragmentation buffer was added to shear mRNA into short fragments and to synthesize the first-strand cDNA with random hexamer primers. The second-strand cDNA was synthesized using buffer, dNTPs, RNaseH, and DNA polymerase I, respectively. Short fragments were purified with QiaQuick PCR extraction kit and resolved with EB buffer for end reparation and poly $(\mathrm{A})$ addition. After that, the short fragments were ligated to sequencing adapters and suitable fragments were selected for the PCR amplification as templates and separated with agarose gel electrophoresis before sequencing. Raw image files were processed by Illumina pipeline for base calling with default parameters resulting in 90 bp paired end reads. Reads with too many $\mathrm{N}$ bases $(>10 \%)$ or low base quality $(>50 \%$ bases with base quality $<5$ ) were discarded. Library construction and sequencing was performed at the Beijing Genomics Institute (BGI), Hong Kong.

\section{Fusion gene analysis}

To achieve robust results, fusion gene discovery was performed using two different strategies. First, we applied ChimeraScan [36] to the raw FASTQ format sequencing data. ChimeraScan used an installed instance of Bowtie 0.12.8 [37] for read alignment. Anchor length was specified as $25 \mathrm{bp}$. One nucleotide mismatch was allowed in the initial alignments and in the alignment of discordant reads. Fusion gene candidates with less than 20 spanning reads were filtered out in order to focus the analysis on strongly expressed fusion genes.

Second, we used an in-house fusion detection algorithm called Breakfast to validate the ChimeraScan results and to search for more complex rearrangement. The Breakfast algorithm operates on aligned SAM files, and therefore we first aligned our whole transcriptome sequencing reads against the GRCh37 human reference genome using Tophat version 2.0.4 [38]. Breakfast searched the alignments for discordant read pairs and unaligned individual mates. For discordant read pairs, we required the mates to be at least one megabase apart. The alignment quality of both mates in a discordant pair was required to be above 15 phred. Next, individual unaligned mates were split into two $25 \mathrm{bp}$ anchors that were extracted from both ends of each $90 \mathrm{bp}$ mate. The $25 \mathrm{bp}$ anchors were then re-aligned against the GRCh37 human reference genome using Bowtie 0.12.8 [37], and the resulting alignments were searched for evidence of discordantly aligned anchor pairs. Breakfast then constructed clusters of evidence for chromosomal rearrangements using both discordant read pairs and anchor pairs. To produce the 
final list of rearrangement candidates, we filtered out any rearrangements that were not supported by at least 1 paired read and 5 anchor pairs, or by at least 20 anchor pairs.

\section{Sanger sequencing of TMPRSS2-SKIL fusion}

The TMPRSS2-SKIL junction was amplified from cDNA using Phusion ${ }^{\mathrm{TM}}$ High-Fidelity DNA polymerase (Finnzymes, Espoo, Finland) and primers 5'-AGTAGGCGCGAGCTAAGCAG-3' (forward) and 5'-CAATGCAATGGTCTGGTTTG-3' (reverse). PCR cycling was performed as follows: $98^{\circ} \mathrm{C}$ for $30-60$ seconds followed by 35 cycles of $98^{\circ} \mathrm{C}$ for 7 seconds, $56-58^{\circ} \mathrm{C}$ for 30 seconds, $72^{\circ} \mathrm{C}$ for 30 seconds, and a final extension for 5 minutes using GC-buffer, with a final volume of $25 \mu 1$. The size of each amplicon was verified with $1 \%$ Agarose gel. Target amplicons were purified using QIAquick PCR purification columns (Qiagen Inc, Valencia, CA, USA) and then sequenced using the BigDye ${ }^{\circledR}$ Terminator v3.1 Cycle Sequencing Kit (Applied Biosystems, Foster City, CA, USA) and the ABI PRISM $® 3100$ sequencer (Applied Biosystems, Foster City, CA, USA) according to manufacturer's instructions.

\section{SKIL expression analysis using qRT-PCR}

cDNA synthesis was performed from $1 \mu \mathrm{g}$ of total RNA using SuperScript(TM) III Reverse Transcriptase (Invitrogen, Carlsbad, CA, USA) and random hexamer primers (Thermo Scientific, Waltham, MA, USA) according to manufacturers' instructions. Synthesized cDNA was diluted 1:20 to nuclease free water and expression was measured using Bio-Rad CFX96 Real Time System. The final reaction mixture $(22 \mu \mathrm{l})$ contained $2 \mu \mathrm{l}$ cDNA, $0.125 \mu \mathrm{l}$ forward (5'-AGAGGCTGAATATGCAGGACA-3') and reverse (5'-CCAAAGCAAGCAACAAACAA-3') primers and $11 \mu 12 X$ SYBR Green qPCR Master Mix and RNAse free ddH2O. NTC (No Template Control) was used during the reaction to detect DNA contamination. PCR cycling was performed as follows: $95{ }^{\circ} \mathrm{C}$ for 20 seconds followed by 55 cycles of $95^{\circ} \mathrm{C}$ for 10 seconds, $58^{\circ} \mathrm{C}$ for 10 seconds, $72^{\circ} \mathrm{C}$ for 8 seconds, followed by melting curve analysis. Finally the size of the amplicon was checked using $1.5 \%$ agarose gel electrophoresis. SKIL expression was normalized relative to the TATA-box binding protein $(T B P)$ reference gene.

\section{Fluorescence in situ hybridization}

Frozen 5-7 $\mu \mathrm{m}$ sections of prostate cancer tissue were fixed by Carnoy fixation (Carnoy fixative: 1/3 acetic acid glacial/methanol, 50\%, 75\%, 2 x 100\%, $15 \mathrm{~min}$ each), denatured in $70 \%$ formamide $/ 2 \times \mathrm{SSC}$ at $72-75^{\circ} \mathrm{C}$ for 3-5 min and air dried. BAC clones were obtained from Life Technologies. Fusion probes (RP11-814F13, upstream of TMPRSS2, and RP11-922G14, overlapping and downstream of $S K I L)$ as well as break-apart probes (CTD-2562E3, upstream of SKIL, and RP11-469J4 downstream of $S K I L)$ were used. The probes were labeled by nick translation with ChromaTide Alexa Fluor 594-5dUPT or digoxigenin-11-dUTP (Roche Diagnostics, Basel, Switzerland). After hybridization for two days at $37^{\circ} \mathrm{C}$ the slides were washed and stained with anti digoxigeninFITC. The slides were embedded in Vectashield antifade solution (Vector Laboratories, Burlingame, CA, USA) containing $0.1 \mathrm{M}$ 4,6-diamidino-2-phenylindole (DAPI) as a counterstain and the signals were scored with an Olympus BX5 epifluorescence microscope equipped with a charge-coupled device camera. Stacks of seven images were captured with each filter set with Image-Pro Plus 6.1 software (Media Cybernetics, Inc., Rockville, MD, USA) and combined to produce an RGB image with an extended depth of focus.

\section{SKIL western blot}

Cells were lysed in RIPA buffer (Thermo Scientific) including protease and phosphatase inhibitors (Halt Protease\&Phosphatase Inhibitor cocktail, Thermo Scientific). Protein concentrations were measured with DC Protein Assay (BioRad), 4x SDS sample buffer was added and $40 \mu \mathrm{g}$ of protein lysate was loaded into each well. SDS-PAGE and protein transfer to nitrocellulose membranes were carried out according to standard protocols. Blocking was achieved with 5\% milk, TBS, 0.1\% Tween. Anti-SnoN (dilution 1:1000, ab128079, Abcam), anti- $\beta$-tubulin I (dilution 1:20000, T7816, SigmaAldrich), and polyclonal HRP-conjugated rabbit antimouse (dilution 1:2000, P0161, Dako) antibodies were used for protein detection.

\section{SKIL immunohistochemistry}

SKIL-protein levels were validated from paraffinembedded prostate cancer tissues using monoclonal mouse antibody (1:700, [2F6] (ab128079), Abcam plc, Cambridge, UK) with Power Vision+ Poly-HRP IHC kit (Immunologic, AD Duiven, the Netherlands) according to the manufacturer's instructions. Prior to staining, sections were deparaffinized and autoclaved in $10 \mathrm{mM}$ of sodium citrate buffer, $\mathrm{pH}$ 6.0. Slides were scanned with an Aperio ScanScope XT scanner (Aperio Technologies, Inc.).

\section{RNA in situ hybridization}

FFPE tissue sections were treated according to manufacturer's instructions using RNAscope ${ }^{\circledR} 2.0 \mathrm{HD}$ 
Detection Kit - BROWN (Advanced Cell Diagnostics, Inc., Hayward, CA, USA). Briefly, slides were first deparaffinized in xylene and dehydrated in 100\% ethanol. Sections were then pretreated and boiled in $50 \mathrm{mM}$ Tris $1 \mathrm{mM}$ EDTA-solution containing $0.05 \%$ Tween using Lab Vision ${ }^{\mathrm{TM}}$ PT Module (Thermo Fisher Scientific Inc., Waltham, MA, USA). Next, a target probe for SKIL mRNA (P/N 427981, Advanced Cell Diagnostics, Inc.) and signal amplifiers were hybridized using HybEZ Oven (Advanced Cell Diagnostics, Inc.). A probe for Peptidylprolyl isomerase B (PPIB, P/N 427981, Advanced Cell Diagnostics, Inc.) was used as a positive control and a probe for dihydrodipicolinate reductase (DapB, P/N 310093, Advanced Cell Diagnostics, Inc.) was used as a negative control in every assay. Signal detection was performed using DAB substrate as a chromogen. Slides were counterstained with 50\% Mayer's hematoxylin (Oy FF-Chemicals $\mathrm{Ab}$ ) and blue color was intensified with TBS-Tween. Finally, slides were dehydrated in an ethanol series and mounted. Slides were scanned with an Aperio ScanScope XT scanner (Aperio Technologies, Inc.).

\section{Small interfering RNA knockdown of SKIL}

The knockdown of SKIL expression was done using small interfering RNA (AM16708, ID 107695) from Ambion (Ambion, Austin, TX, USA). The siRNA sequences (5'-to-3') were:

1: GGCAAGUAAGUCCAUAUCATT (sense) and UGAUAUGGACUUGCCTC (antisense)

2: GGCUCACAGUAGUGGUAATT (sense) and UUACCACUACUGUGAGCCTT (antisense)

Silencer® Negative Control \#1 siRNA (AM4611, Ambion, Austin, TX, USA) was used as a non-targeting control. Cells were seeded into 24 -well plates (30000 cells/ well) in four replicates and were transfected the following day with $50 \mathrm{nM}$ SKIL siRNA and scrambled siRNA. INTERFERin TM (PolyPlus Transfection, Strasbourg, France) and Opti-MEM were used for cell transfection.

\section{Growth curve analysis}

Cells were plated on 12-well plate (30 000 cells on each well) as quadruplicates and each well was scanned daily using the Surveyor Software (Objective Imaging Ltd.) with a camera (Imaging Inc., Canada) attached to the Olympus IX71 (Olympus, Tokyo, Japan) microscope and the area of the attached cells in each well was computed using ImageJ Software (Wayne Rasband, National Institutes of Health, Bethesda, MD) and divided by the mean area for day 1 .

\section{Cell invasion assay}

The effect of SKIL knockdown in PC-3 cells and overexpression in RWPE-1 cells on cell invasion were evaluated in BioCoat Matrigel Invasion chambers (BD Biosciences, Bedford, MA, USA) coated with a basement membrane matrix. Matrigel was rehydrated in growth medium for $2 \mathrm{~h}$ at $37^{\circ} \mathrm{C}$ and $5 \% \mathrm{CO} 2$. Transfected cells (10,000 PC-3 or 20,000 RWPE-1) were harvested and resuspended in 1\% FBS (PC-3 cells) or Keratinocyte Serum Free Medium (RWPE-1 cells), and placed in the upper chamber of the transwell. The lower chamber contained a $750 \mu \mathrm{l}$ medium with $10 \% \mathrm{FBS}$ and $5 \mu \mathrm{g} / \mathrm{ml}$ fibronectin (PC-3 cells) or $0.05 \mathrm{mg} / \mathrm{ml} \mathrm{BPE}$ and $5 \mathrm{ng} / \mathrm{ml}$ EGF (RWPE-1 cells). The cells were then incubated for $22 \mathrm{~h}$ at $37{ }^{\circ} \mathrm{C}$ in $5 \% \mathrm{CO}$. Cells in the top well of the upper chamber were wiped off the top membrane with cotton swabs. The membranes were then fixed with 3.7\% formaldehyde (PC-3 cells) or 100\% methanol (RWPE-1 cells) and stained with $1 \%$ toluidine for $15 \mathrm{~min}$ at room temperature for visualization of cells. Cells that had invaded to the lower surface were photographed and counted under a microscope.

\section{Colony formation assay}

PC-3 cells transfected with SKIL or scrambled siRNA were grown on 6-well plates in duplicate (5000 cells/well). Briefly, base agar containing 1\% agar dissolved in Ham's F-12 mixed with 20\% FBS, 2\% L-glutamine and $2 \%$ penicillin-streptomycin and $1 \mathrm{ml}$ mixture was transferred into the well. Then the top layer containing $0.7 \%$ agar including $10 \% \mathrm{FBS}, 1 \%$ L-glutamine and $1 \%$ penicillin-streptomycin was prepared. Top agar was mixed with suspended cell line and transferred over base agar. Base and top agar were covered with normal growth medium and incubated at $37^{\circ} \mathrm{C}$ and $5 \% \mathrm{CO} 2$ for 15 days. After incubation, colonies were fixed with $3.7 \%$ formaldehyde and stained with $0.1 \%$ toluidine blue. Excess dye was removed by washing with $10 \mathrm{mM}$ phosphate buffer ( $\mathrm{pH}$ 7.4).

\section{Transfection of SKIL into RWPE-1 cells}

To study the effects of SKIL overexpression, pCINeo HA-hSnoN plasmid (a gift from Robert Weinberg, Addgene plasmid \#10908 [17]) and universal empty, pCI-Neo backbone pUNIV-plasmid (a gift from Cynthia Czajkowski, Addgene plasmid \#24705 [39]) were stably transfected into RWPE-1 cells (ATCC, cultured under the recommended conditions) with jetPEI ${ }^{\circledR}$ polymer-based DNA transfection reagent (POLYPLUSTRANSFECTION Inc., New York, NY, USA) according to the manufacturer's instructions. Transfected clones were 
selected with $400 \mu \mathrm{g} / \mathrm{ml}$ geneticin (G418, Invitrogen Inc., Carlsbad, CA, USA) over several weeks, after which SKIL mRNA levels were determined using qRT-PCR and the clone showing the highest overexpression of SKIL mRNA was selected for further analysis. Control RWPE-1 cells were transfected with empty pCI-Neo backbone vector. All transfected cells were subsequently cultured after selection in a medium containing geneticin $(200 \mu \mathrm{g} / \mathrm{ml})$.

\section{Calculation of gene expression}

RNA-seq reads were aligned against RefSeq 38 human transcript sequences using Bowtie version 2.0.0beta6 [40]. Expression values were normalized across all samples using median-of-ratios normalization. Read counts for a given gene were divided by the total length of the gene's exons (in kilobases) to correct for gene size bias. For some genes, we observed a strong and systematic expression bias associated with the two different RNA isolation methods we used (Trizol and Qiagen AllPrep kits). To correct for this bias, we took the BPH samples from which we had extracted RNA using both Trizol and Qiagen, and calculated an expression ratio for all genes by dividing the median expression in the Trizol group by the median expression in the Qiagen group. We then used an unpaired t-test to look for genes differentially expressed between Trizol and Qiagen treated samples. Genes with a p-value less than 0.0001 were considered sensitive to the RNA isolation method, and their expression levels were corrected by dividing the expression of Trizol samples with the Trizol/Qiagen expression ratio.

\section{Copy number analysis}

DNA-seq reads were aligned against GRCh37 using Bowtie version 2.0.0-beta6 [40]. Aligned read counts were calculated within overlapping $500 \mathrm{bp}$ windows along the whole genome. Coverage logratios were calculated within each window by comparing against read count averages from four BPH controls. To normalize logratios within copy neutral regions to zero, we applied a median filter of length 50 to the logratios in each sample, rendered logratio histograms for each chromosome, and took the median of the histogram modes. This value was then subtracted from all logratios for that sample. Frequently aberrant chromosomes 8, 22, X, and Y were not included in calculating the median of modes. Coverage logratios for individual genes were calculated by taking the median logratio over all intragenic windows. If a gene's length was shorter than $20 \mathrm{~kb}$, the median window was extended on both sides so as to reach a length of $20 \mathrm{~kb}$. Logratios were converted into copy number changes using the formula (ploidy $* 2^{\wedge} \operatorname{logratio}$ - ploidy), where ploidy was based on the chromosome in which the gene or genomic region was located. Copy number changes were further multiplied by
(1 / 0.7) to correct for the estimated $70 \%$ tumor sample purity in our samples. A gene was considered to be amplified or deleted if the corrected copy number change had an absolute value above 0.5 .

\section{Computational identification of putative somatic mutations}

We first used RNA-seq data to search for mutations in transcribed loci, and then validated putative variants using DNA-seq data. RNA-seq reads were aligned against GRCh37 using Tophat version 2.0.2 [38] and Bowtie version 2.0.0-beta6 [40]. Duplicate reads were discarded using samtools rmdup, and variants were called using an in-house pipeline. Low quality alignments (MAPQ $<10)$ were ignored for PC and CRPC samples, but were used for variant calling in $\mathrm{BPH}$ control samples. A genomic site was called heterozygous alternate in PC and CRPC if at least four reads and at least $15 \%$ of all reads at that site showed an alternate allele. In BPH samples, a genomic site was considered heterozygous if at least 2 reads and at least $5 \%$ of all reads showed an alternate allele. Any variants found in BPH samples were considered to be germline variants and filtered out. We also filtered out any variants found in the 1000 Genomes project [41], the NHLBI Exome Sequencing Project (ESP6500, unpublished, http://evs.gs.washington.edu/EVS/), or the SISU project (unpublished, http://www.sisuproject.fi/). The VCF file produced by samtools was annotated using the ANNOVAR software [42] and custom scripts. For every variant, we predicted functional impact and identified features in the genomic neighborhood. We also determined whether the variant was found in the COSMIC database [43] for cancer associated mutations.

\section{Illumina MiSeq targeted validation of somatic mutations}

Sequencing libraries were prepared using the the Illumina TruSeq Custom Amplicon Kit following the TruSeq Custom Amplicon Library Preparation Guide. For most samples, $250 \mathrm{ng}$ of DNA was used. Custom probes flanking 677 target variants were designed using the DesignStudio software (Illumina). Probes were designed so as to yield amplicons with a mean size of 250 bp. Targets were extended from one probe and ligated to the second probe. Next, during two rounds of synthesis, sample-specific indexes were incorporated, producing dsDNA molecules containing two unique indexes and flanking amplification sequences. Libraries were then generated using the following PCR program: $95^{\circ} \mathrm{C}$ for 180 seconds, 24 cycles of $95^{\circ} \mathrm{C}$ for 30 seconds, $66^{\circ} \mathrm{C}$ for 30 seconds, $72^{\circ} \mathrm{C}$ for 60 seconds, and $72^{\circ} \mathrm{C}$ for 5 minutes, then cooling to $10^{\circ} \mathrm{C}$. Resulting libraries were cleaned up ( $45 \mu \mathrm{l}$ of AMPure XP beads for each library) 
and normalized according to manufacturer's guidelines. Library quality was verified with an Agilent Bioanalyzer (Agilent Santa Clara, CA, USA) using a DNA 1000 chip. Finally, all libraries were pooled and diluted 1:100 before loading into a MiSeq instrument (Illumina, San Diego, CA, USA) for sequencing. The reagent kit used was the MiSeq ${ }^{\circledR}$ Reagent Kit v2 (500 cycle).

\section{Data access}

The European Genome-phenome archive database accession number for the high throughput sequencing data reported in this paper is EGAS00001000526.

\section{ACKNOWLEDGEMENTS}

The work was supported by grants from the Finnish Funding Agency for Technology and Innovation Finland Distinguished Professor programme (OYH, MN), Academy of Finland (project no. 269474 MN, project no. 251790 OYH, project no. 127187 TV), Sigrid Juselius Foundation (MN, TV), Emil Aaltonen Foundation (MA), Cancer Society of Finland (MN, TV), Competitive State Research Financing of the Expert Responsibility area of Tampere University Hospital (Grant 9P053 TLJT, Grant 9N087 TV), and EU-FP7 Marie Curie Integrated Training Network, PRO-NEST (TV), the National Institutes of Health (U24CA143835, WZ). We thank Paula Kosonen, Riina Kylätie, Päivi Martikainen, Marika VähäJaakkola, Katja Liljeström and Mariitta Vakkuri for their technical assistance and Patholog Teemu Tolonen for his professional assistance.

The results published here are in part based upon data generated by The Cancer Genome Atlas project (dbGaP Study Accession: phs000178.v8.p7) established by the NCI and NHGRI. Information about TCGA and the investigators and institutions who constitute the TCGA research network can be found at http://cancergenome.nih. gov.

\section{Conflicts of interest}

The authors declare no conflicts of interest.

\section{Author contributions}

MA performed computational identification of fusion genes, mutations and copy number alterations. KK validated TMPRSS2-SKIL using Sanger sequencing. SK, KK, JT and LL performed SKIL siRNA experiments. JT and KK performed SKIL overexpression experiments. KK, JT and SK performed qRT-PCR experiments. KG and LL performed western blot experiments. OS performed FISH experiments. LS performed RNA in situ experiments. KK and KMK validated mutations using MiSeq and Sanger sequencing. PR performed image analysis. MA and KK performed statistical analyses. AY, OYH, WZ, TV and MN conceived of the study. MA, KK, AY, TV and MN wrote the manuscript. RLV and TLJT provided materials and participated in design and coordination. All authors read and approved the manuscript.

\section{REFERENCES}

1. Jemal A, Bray F, Center MM, Ferlay J, Ward E, Forman D. Global cancer statistics. CA Cancer J. Clin. 2011;61(2):6990.

2. Wong YNS, Ferraldeschi R, Attard G, de Bono J. Evolution of androgen receptor targeted therapy for advanced prostate cancer. Nat. Rev. Clin. Oncol. 2014;11(6):365-76.

3. Taylor BS, Schultz N, Hieronymus H, Gopalan A, Xiao Y, Carver BS, Arora VK, Kaushik P, Cerami E, Reva B, Antipin Y, Mitsiades N, Landers T, et al. Integrative genomic profiling of human prostate cancer. Cancer Cell 2010;18(1):11-22.

4. Barbieri CE, Baca SC, Lawrence MS, Demichelis F, Blattner M, Theurillat J-P, White TA, Stojanov P, Van Allen E, Stransky N, Nickerson E, Chae S-S, Boysen G, et al. Exome sequencing identifies recurrent SPOP, FOXA1 and MED12 mutations in prostate cancer. Nat. Genet. 2012;44(6):685-9.

5. Tomlins SA, Rhodes DR, Perner S, Dhanasekaran SM, Mehra R, Sun X-W, Varambally S, Cao X, Tchinda J, Kuefer R, Lee C, Montie JE, Shah RB, et al. Recurrent fusion of TMPRSS2 and ETS transcription factor genes in prostate cancer. Science 2005;310(5748):644-8.

6. Kumar-Sinha C, Tomlins SA, Chinnaiyan AM. Recurrent gene fusions in prostate cancer. Nat. Rev. Cancer 2008;8(7):497-511.

7. Tomlins SA, Rhodes DR, Yu J, Varambally S, Mehra R, Perner S, Demichelis F, Helgeson BE, Laxman B, Morris DS, Cao Q, Cao X, Andrén O, et al. The role of SPINK1 in ETS rearrangement-negative prostate cancers. Cancer Cell 2008;13(6):519-28.

8. Ateeq B, Tomlins SA, Laxman B, Asangani IA, Cao Q, Cao X, Li Y, Wang X, Feng FY, Pienta KJ, Varambally S, Chinnaiyan AM. Therapeutic targeting of SPINK1-positive prostate cancer. Sci. Transl. Med. 2011;3(72):72ra17.

9. Burkhardt L, Fuchs S, Krohn A, Masser S, Mader M, Kluth M, Bachmann F, Huland H, Steuber T, Graefen M, Schlomm T, Minner S, Sauter G, et al. CHD1 is a $5 \mathrm{q} 21$ tumor suppressor required for ERG rearrangement in prostate cancer. Cancer Res. 2013;73(9):2795-805.

10. Grasso CS, Wu Y-M, Robinson DR, Cao X, Dhanasekaran SM, Khan AP, Quist MJ, Jing X, Lonigro RJ, Brenner JC, Asangani IA, Ateeq B, Chun SY, et al. The mutational landscape of lethal castration-resistant prostate cancer. Nature 2012;487(7406):239-43. 
11. Ding Z, Wu C-J, Chu GC, Xiao Y, Ho D, Zhang J, Perry SR, Labrot ES, Wu X, Lis R, Hoshida Y, Hiller D, Hu $\mathrm{B}$, et al. SMAD4-dependent barrier constrains prostate cancer growth and metastatic progression. Nature 2011;470(7333):269-73.

12. Qin J, Wu S-P, Creighton CJ, Dai F, Xie X, Cheng C-M, Frolov A, Ayala G, Lin X, Feng X-H, Ittmann MM, Tsai S-J, Tsai M-J, et al. COUP-TFII inhibits TGF- $\beta$-induced growth barrier to promote prostate tumorigenesis. Nature 2013;493(7431):236-40.

13. Bjerke GA, Pietrzak K, Melhuish TA, Frierson HF Jr, Paschal BM, Wotton D. Prostate cancer induced by loss of Apc is restrained by TGF $\beta$ signaling. PLoS One 2014;9(3):e92800.

14. Aitchison AA, Veerakumarasivam A, Vias M, Kumar R, Hamdy FC, Neal DE, Mills IG. Promoter methylation correlates with reduced Smad4 expression in advanced prostate cancer. Prostate 2008;68(6):661-74.

15. Hahn SA, Schutte M, Hoque AT, Moskaluk CA, da Costa LT, Rozenblum E, Weinstein CL, Fischer A, Yeo CJ, Hruban RH, Kern SE. DPC4, a candidate tumor suppressor gene at human chromosome 18q21.1. Science 1996;271(5247):350-3.

16. Stroschein SL, Wang W, Zhou S, Zhou Q, Luo K. Negative feedback regulation of TGF-beta signaling by the SnoN oncoprotein. Science 1999;286(5440):771-4.

17. Sun Y, Liu X, Ng-Eaton E, Lodish HF, Weinberg RA. SnoN and Ski protooncoproteins are rapidly degraded in response to transforming growth factor beta signaling. Proc. Natl. Acad. Sci. U. S. A. 1999;96(22):12442-7.

18. Cho N-Y, Choi M, Kim B-H, Cho Y-M, Moon KC, Kang GH. BRAF and KRAS mutations in prostatic adenocarcinoma. Int. J. Cancer 2006;119(8):1858-62.

19. Lindhurst MJ, Sapp JC, Teer JK, Johnston JJ, Finn EM, Peters K, Turner J, Cannons JL, Bick D, Blakemore L, Blumhorst C, Brockmann K, Calder P, et al. A Mosaic Activating Mutation in AKT1 Associated with the Proteus Syndrome. N. Engl. J. Med. 2011;365(7):611-9.

20. Ewing CM, Ray AM, Lange EM, Zuhlke KA, Robbins CM, Tembe WD, Wiley KE, Isaacs SD, Johng D, Wang Y, Bizon C, Yan G, Gielzak M, et al. Germline Mutations in HOXB13 and Prostate-Cancer Risk. N. Engl. J. Med. 2012;366(2):141-9.

21. Ramos-Montoya A, Lamb AD, Russell R, Carroll T, Jurmeister S, Galeano-Dalmau N, Massie CE, Boren J, Bon H, Theodorou V, Vias M, Shaw GL, Sharma NL, et al. HES6 drives a critical AR transcriptional programme to induce castration-resistant prostate cancer through activation of an E2F1-mediated cell cycle network. EMBO Mol. Med. 2014;6(5):651-61.

22. Annala M, Kivinummi K, Leinonen K, Tuominen J, Zhang W, Visakorpi T, Nykter M. DOT1L-HES6 fusion drives androgen independent growth in prostate cancer. EMBO Mol. Med. 2014;6(9):1121-3.
23. Nomura N, Sasamoto S, Ishii S, Date T, Matsui M, Ishizaki R. Isolation of human cDNA clones of ski and the skirelated gene, sno. Nucleic Acids Res. 1989;17(14):5489500 .

24. Pearson-White S. Snol, a novel alternatively spliced isoform of the ski protooncogene homolog, sno. Nucleic Acids Res. 1993;21(19):4632-8.

25. Li Y, Turck CM, Teumer JK, Stavnezer E. Unique sequence, ski, in Sloan-Kettering avian retroviruses with properties of a new cell-derived oncogene. J. Virol. 1986;57(3):1065-72.

26. Boyer PL, Colmenares C, Stavnezer E, Hughes SH. Sequence and biological activity of chicken snoN cDNA clones. Oncogene 1993;8(2):457-66.

27. Deheuninck J, Luo K. Ski and SnoN, potent negative regulators of TGF- $\beta$ signaling. Cell Res. 2008;19(1):47-57.

28. Hagerstrand D, Tong A, Schumacher SE, Ilic N, Shen RR, Cheung HW, Vazquez F, Shrestha Y, Kim SY, Giacomelli AO, Rosenbluh J, Schinzel AC, Spardy NA, et al. Systematic interrogation of 3q26 identifies TLOC1 and SKIL as cancer drivers. Cancer Discov. 2013;3(9):1044-57.

29. Wu JW, Krawitz AR, Chai J, Li W, Zhang F, Luo K, Shi Y. Structural mechanism of Smad4 recognition by the nuclear oncoprotein Ski: insights on Ski-mediated repression of TGF-beta signaling. Cell 2002;111(3):357-67.

30. Band AM, Laiho M. SnoN oncoprotein enhances estrogen receptor- $\alpha$ transcriptional activity. Cell. Signal. 2012;24(4):922-30.

31. Dubbink HJ, Hersmus R, Pike ACW, Molier M, Brinkmann AO, Jenster G, Trapman J. Androgen receptor ligandbinding domain interaction and nuclear receptor specificity of FXXLF and LXXLL motifs as determined by L/F swapping. Mol. Endocrinol. 2006;20(8):1742-55.

32. Maher CA, Kumar-Sinha C, Cao X, Kalyana-Sundaram S, Han B, Jing X, Sam L, Barrette T, Palanisamy N, Chinnaiyan AM. Transcriptome sequencing to detect gene fusions in cancer. Nature 2009;458(7234):97-101.

33. Bolton NJ, Lahtonen R, Vihko P, Kontturi M, Vihko R. Androgens and prostate-specific acid phosphatase in whole tissue and in separated epithelium from human benign prostatic hypertrophic glands. Prostate 1981;2(4):409-16.

34. Cerveira N, Ribeiro FR, Peixoto A, Costa V, Henrique R, Jerónimo C, Teixeira MR. TMPRSS2-ERG gene fusion causing ERG overexpression precedes chromosome copy number changes in prostate carcinomas and paired HGPIN lesions. Neoplasia 2006;8(10):826-32.

35. Corey E, Vessella RL. Xenograft models of human prostate cancer. In: Prostate cancer. Springer; 2007. page 3-31.

36. Iyer MK, Chinnaiyan AM, Maher CA. ChimeraScan: A tool for identifying chimeric transcription in sequencing data. Bioinformatics 2011;27(20):2903-4.

37. Langmead B, Trapnell C, Pop M, Salzberg SL. Ultrafast and memory-efficient alignment of short DNA sequences to the human genome. Genome Biol. 2009;10(3):R25. 
38. Kim D, Pertea G, Trapnell C, Pimentel H, Kelley R, Salzberg S. TopHat2: accurate alignment of transcriptomes in the presence of insertions, deletions and gene fusions. Genome Biol. 2013;14(4):R36.

39. Venkatachalan SP, Bushman JD, Mercado JL, Sancar F, Christopherson KR, Boileau AJ. Optimized expression vector for ion channel studies in Xenopus oocytes and mammalian cells using alfalfa mosaic virus. Pflugers Arch. 2007;454(1):155-63.

40. Langmead B, Salzberg SL. Fast gapped-read alignment with Bowtie 2. Nat. Methods 2012;9(4):357-9.

41. The 1000 Genomes Project Consortium. A map of human genome variation from population-scale sequencing. Nature 2010;467(7319):1061-73.

42. Wang K, Li M, Hakonarson H. ANNOVAR: functional annotation of genetic variants from high-throughput sequencing data. Nucleic Acids Res. 2010;38(16):e164.

43. Bamford S, Dawson E, Forbes S, Clements J, Pettett R, Dogan A, Flanagan A, Teague J, Futreal PA, Stratton MR, Wooster R. The COSMIC (Catalogue of Somatic Mutations in Cancer) database and website. Br. J. Cancer 2004;91(2):355-8. 